\title{
Reprodutibilidade de um teste microbiológico para estreptococos do grupo mutans
}

\section{Reproducibility of a simplified microbiological test for mutans streptococci}

\author{
Camila PINELLI* \\ Leonor de Castro Monteiro LOFFREDO** \\ Mônica Campos SERRA***
}

\begin{abstract}
PINELLI, C.; LOFFREDO, L. C. M.; SERRA, M. C. Reprodutibilidade de um teste microbiológico para estreptococos do grupo mutans. Pesq Odont Bras, v. 14, n. 1, p. 13-18, jan./mar. 2000.

Com o desenvolvimento de testes microbiológicos salivares simplificados tornou-se mais prático o diagnóstico de pacientes com baixa atividade, porém com alto risco de cárie. O propósito desta pesquisa foi avaliar a reprodutibilidade do CARITEST-SM ${ }^{\circledR}$, um teste específico para estreptococos do grupo mutans. Catorze voluntários participaram do estudo. A produção de saliva foi estimulada com uma goma de mascar inerte e coletada até a obtenção do volume suficiente para a execução de dois testes - pertencentes a dois conjuntos de lotes diferentes de "kits" CARITEST-SM ${ }^{\circledR}$ (1 e 2) aplicados para um mesmo indivíduo. Dois examinadores calibrados realizaram a leitura de forma independente e cega, classificando os resultados de duas maneiras: escores de 1 a 6 , conforme recomenda o fabricante, e conforme a classificação quanto à contagem microbiológica, baixa e alta. Foi aplicada a estatística Kappa, segundo Light. Os resultados de concordância, conforme 6 escores, mostraram valores distintos para o conjunto dos "kits" 1 e para o conjunto dos "kits" 2, valendo, respectivamente, $\kappa=0,57$ e $\kappa=0,21$. Em relação à classificação conforme a contagem microbiológica, alta e baixa, os resultados foram $\kappa=1,00$, para o conjunto dos "kits" 1 , e $\kappa=0,55$, para o conjunto dos "kits" 2. Assim, estes índices de concordância mostraram que a diferença no lote exerceu uma variabilidade no resultado. Além disso, houve maior concordância interexaminador quando se utilizou a classificação conforme a contagem microbiológica alta e baixa.
\end{abstract}

UNITERMOS: Testes microbiológicos; Saliva; Cárie dentária.

\section{INTRODUÇÃO}

A cárie dental é uma patologia que tem início antes do desenvolvimento da lesão clinicamente detectável ${ }^{4}$. Sua manifestação provém de uma etiologia multifatorial na qual interagem superficie dentária, carboidratos da dieta - principalmente a sacarose - saliva e os microrganismos da placa bacteriana ${ }^{5,10}$. Entretanto, nem todos os indivíduos de uma determinada população apresentam predisposições semelhantes para o desenvolvimento de cáries. A variabilidade individual do risco de cárie está relacionada, principalmente, com a dieta consumida e com o número de microrganismos cariogênicos presentes na placa bacteriana e na saliva ${ }^{23}$.

Diversas tentativas têm sido feitas para se utilizar bactérias bucais ou seus produtos para a predição do risco de cárie em humanos ${ }^{22}$. As prin- cipais bactérias associadas à predição do risco de cárie são os lactobacilos e os estreptococos do grupo mutans ${ }^{21,22}$.

Testes bacteriológicos foram introduzidos na Odontologia, na década de 50, quando, através do Ágar Rogosa, foi possivel realizar a contagem de lactobacilos na saliva. Com relação aos estreptococos do grupo mutans, puderam ser quantificados através do ágar mitis salivarius, acrescido do antibiótico bacitracina e de uma concentração mais alta de sacarose, o M.S.B. ${ }^{6}$. O método clássico, para a contagem de estreptococos do grupo $\mathrm{mu}$ tans $^{6}$, consiste na semeadura de diferentes diluições de saliva em várias placas de ágar M.S.B., incubação em anaerobiose a $37^{\circ} \mathrm{C}$ e leitura em microscópio estereoscópico. Maior praticidade surgiu com método da espátula de madeira, pro-

\footnotetext{
*Doutoranda em Clínica Odontológica; *** Professora Doutora do Departamento de Odontologia Restauradora - Faculdade de Odontologia da Universidade de Campinas - Piracicaba.

** Professora Doutora do Departamento de Odontologia Social da Faculdade de Odontologia da Universidade Estadual Paulista Araraquara.
} 
PINELLI, C.; LOFFREDO, L. C. M.; SERRA, M. C. Reprodutibilidade de um teste microbiológico para estreptococos do grupo mutans.

Pesq Odont Bras, v. 14, n. 1, p. 13-18, jan./mar. 2000.

posto por KÖHLER e BRATTHALL ${ }^{13}$, em 1979. Embora abolisse as diluições, não eliminou a necessidade do ágar M.S.B., restringindo o seu uso, devido à vida útil pequena, de uma semana, após preparado. Outras tentativas foram propostas para a obtenção de métodos mais práticos de aplicação clínica, envolvendo o mínimo de treinamento laboratorial e vida útil mais longa ${ }^{2,8,9,18,20}$. Em 1987, JORDAN et al. ${ }^{9}$ desenvolveram um teste salivar simplificado, para estreptococos do grupo $\mathrm{mu}$ tans, em lâmina revestida com ágar mitis salivarius. Nesse teste a bacitracina é adicionada a um frasco contendo solução tamponada, antes da adição de saliva. A lâmina revestida com o ágar é mergulhada na solução e depois transferida para um outro tubo, para a incubação em presença de um tablete gerador de gás carbônico com duas gotas de água. Após 48 horas a $37^{\circ} \mathrm{C}$, o frasco é mantido por mais 24 horas em temperatura ambiente. Então, é feita a avaliação do crescimento, comparando-se a lâmina de ágar com uma escala padrão, fornecida pelo fabricante.

O nivel salivar de estreptococos do grupo $m u$ tans tem sido utilizado como um indicador do risco de cárie, correlacionando-se tanto à experiência passada de cárie ${ }^{11,15,20,21}$, quanto à futura atividade da doença $a^{3,7,14}$.

No Brasil, um teste para estreptococos do grupo mutans semelhante ao desenvolvido por JORDAN et al. ${ }^{9}, 1987$, foi colocado à venda no ano de 1993 (CARITEST SM ${ }^{\circledR}$ - HERPO/Produtos Dentários Ltda.). Um dos problemas que se apresenta é que todo teste diagnóstico deve ser avaliado do ponto de vista de sua qualidade, ou seja, de sua capacidade de produzir informação segura. Assim, torna-se bastante oportuno que seja feito um estudo sobre um aspecto de qualidade do teste: a reprodutibilidade, isto é, a consistência da informação quando o teste se repete. É interessante saber se há concordância entre examinadores, ao atribuírem um diagnóstico de forma independente. Dada a possibilidade de uso do produto em pesquisas epidemiológicas bem como na prática odontológica privada, julgou-se de importância analisar a reprodutibilidade, verificando-se a concordância interexaminador, após uma calibração prévia.

Objetivo: analisar os niveis de concordância interexaminador, utilizando dois lotes diferentes do produto CARITEST SM ${ }^{\circledR}$, na leitura dos resultados microbiológicos, segundo duas classificações:

1. conforme 6 escores, seguindo a recomendação do fabricante, e

2. conforme a contagem microbiológica: baixa contagem (escores 1, 2 e 3 ) e alta contagem (escores 4,5 e 6$)$.

\section{MATERIAL E MÉTODOS}

Para se realizar o teste microbiólogico CARITEST SM ${ }^{\circledR}$, foram selecionados, na Clínica da Faculdade de Odontologia de Piracicaba/ UNICAMP, dezesseis voluntários: oito do sexo feminino e oito do sexo masculino, com idade entre 9 e 13 anos. Catorze completaram o estudo. Os voluntários foram agendados e avisados para não ingerirem alimento ou escovarem os dentes, uma hora antes da realização do teste.

Cada "kit" CARITEST SM ${ }^{\circledR}$ apresenta material para a realização de testes salivares em 5 indivíduos. Contém gomas sem sabor, tubos com diluente tamponado, tubos com lâmina revestida com meio de cultura ágar mitis salivarius, envelopes com bacitracina, envelopes contendo mistura geradora de gás carbônico e uma escala com 6 densidades de crescimento das unidades formadoras de colônia (UFC), 10.000, 50.000, 100.000, 250.000, 500.000 e 1.000 .000 , por mililitro de saliva.

Os indivíduos foram instruídos a mastigar uma goma, vigorosamente, engolindo a saliva produzida nos primeiros 20 segundos. Em seguida, mastigaram e verteram toda a saliva produzida, durante um tempo médio de 15 minutos, num copo plástico, obtendo-se o volume suficiente para a execução de dois testes, para cada participante.

Os voluntários foram dispensados, os recipientes com a saliva foram cobertos com filme de PVC, transportados ao laboratório e utilizados no prazo máximo de 30 minutos após a coleta. Então, os testes foram realizados, com a temperatura ambiente controlada entre 20 e $22^{\circ} \mathrm{C}$. A saliva produzida foi usada para a execução de dois testes de lotes diferentes (conjunto dos "kits" 1 e conjunto dos "kits" 2) para um mesmo indivíduo.

Para um melhor entendimento do texto utilizaram-se os códigos:

- Tubo A-1: tubo contendo diluente tamponado, pertencente ao conjunto dos "kits" 1.

- Tubo B-1: tubo com lâmina de cultura, pertencente ao conjunto dos "kits" 1.

- Tubo A-2: tubo contendo diluente tamponado, pertencente ao conjunto dos "kits" 2.

- Tubo B-2: tubo com lâmina de cultura, pertencente ao conjunto dos "kits" 2.

O primeiro teste foi realizado, acrescentando-se a bacitracina fornecida pelo fabricante (bacitracina USP e aglutinante inerte) e 1,5 $\mathrm{ml}$ de saliva, ao tubo A-1, que foi fechado e gentilmente agitado, durante 10 segundos, para se homogeneizar a 
PINELLI, C.; LOFFREDO, L. C. M.; SERRA, M. C. Reprodutibilidade de um teste microbiológico para estreptococos do grupo mutans.

Pesq Odont Bras, v. 14, n. 1, p. 13-18, jan./mar. 2000.

mistura. A lâmina com meio de cultura, que estava acondicionada no tubo B-1, foi retirada e mergulhada no tubo A-1, fechando-o em seguida. Toda a superficie do meio de cultura foi molhada pela solução com saliva. Enquanto isso, no tubo B-1, que ficou vazio após a remoção da lâmina, colocou-se a mistura geradora de gás carbônico, e duas gotas de água destilada, medidas com conta-gotas. Imediatamente, a lâmina foi removida do tubo A-1, retirando o excesso da solução, e inserida novamente no tubo B-1, que foi fechado e colocado em estufa a $37^{\circ} \mathrm{C}$, por 48 horas. Os mesmos procedimentos, descritos para um tubo do conjunto dos "kits" 1, foram realizados, pelo mesmo operador, simultaneamente para o outro tubo do conjunto dos "kits" 2, durante o tempo de 5 minutos totalizando os 2 testes para cada voluntário. Após as 48 horas, os tubos foram mantidos em estufa, numa temperatura de $23 \pm 1^{\circ} \mathrm{C}$ durante 24 horas. Decorrido este período, procedeu-se à leitura dos resultados. Para a aplicação dos testes, seguiram-se as instruções do fabricante, com a padronização dos procedimentos: a) medição do volume de 1,5 $\mathrm{ml}$ de saliva, com pipeta automática; b) uso de água destilada; c) controle da temperatura ambiente em $23 \pm 1^{\circ} \mathrm{C}$, durante 24 horas.

\section{Avaliação dos resultados microbiológicos}

Dois examinadores, ambos cirurgiões-dentistas, se submeteram a uma calibração prévia, a fim de fornecer as condições necessárias, para a conversão da aparência da lâmina incubada em números correspondentes às densidades de crescimento (UFC/ml saliva). Na sessão de calibração, foi utilizado o quadro de densidade das colônias fornecido pelo fabricante, que apresentava 6 niveis de classificação das UFC: 10.000 / 50.000 / 100.000 / 250.000 / 500.000 e 1.000 .000 , por mililitro de saliva.

Os 14 testes foram avaliados, seguindo as recomendações do fabricante, pela observação do lado da lâmina, com a maior densidade de crescimento. As avaliações foram cegas, já que nenhum examinador teve contato com os voluntários do estudo em momento algum, e realizadas de forma independente: enquanto o examinador 1 lia e anotava os resultados de um teste do lote 1 , o examinador 2 avaliava o teste do lote 2, ambos pertencentes ao mesmo indivíduo ${ }^{4}$. Em seguida, o examinador 1 avaliava o resultado do teste do lote 2 , enquanto o examinador 2 examinava o teste do lote 1 .

Os resultados foram anotados, em fichas apropriadas, conforme duas classificações: em escores de 1 a 6 e, posteriormente, quanto à contagem microbiológica baixa (escores de 1 a 3) e alta (escores de 4 a 6). A linha que divide os escores 3 e 4 (100.000 - 250.000 UFC/ml) da escala de densidades representa, em termos de contagem microbiológica, uma alta infecção por estreptococos do grupo mutans, evidenciando um alto risco de cárie ${ }^{9}$.

\section{Planejamento estatístico}

Para a análise da reprodutibilidade interexaminador foi aplicada a estatística kappa $(\kappa)$, segundo LIGHT $^{17}$ que permitiu a obtenção de concordância por ponto, sendo:

$\kappa=(p o-p e) /(1-p e)$, onde po é a proporção observada de concordância (diagonal principal das Tabelas 2, 3, 4 e 5) e pe é a proporção esperada de concordância. O nivel de significância adotado foi de $5 \%$ para a tomada de decisão.

O padrão de classificação de concordância seguiu o critério proposto por LANDIS; $\mathrm{KOCH}^{16}(\mathrm{Ta}-$ bela 1), admitindo-se a aproximação normal para a variável $z=\kappa / s \kappa$.

\section{RESULTADOS}

Obteve-se um valor de kappa significativo $(\kappa=0,57)$ que, dentro do padrão de classificação proposto, representa uma concordância regular para o conjunto dos "kits" 1 (Tabela 2). Para o conjunto dos "kits" 2, obteve-se um valor de $\kappa=0,21$ (Tabela 3), sendo indicativo de concordância sofrível, embora seja um valor significativo ao nível de $5 \%$. Os resultados de reprodutibilidade interexaminador obtidos foram distintos para os "kits" utilizados, sendo que o conjunto dos "kits" 1 levou a resultados mais favoráveis.

Para a classificação segundo a contagem microbiológica alta e baixa, ao ser avaliado o conjunto dos "kits" 1, observou-se 100\% de concordância interexaminador $(\kappa=1,00)$, ou seja, houve concordância ótima (Tabela 4). Quando se avaliou o conjunto dos "kits" 2, observou-se $\kappa=0,55$, indicativo de uma concordância regular (Tabela 5).

TABELA 1 - Interpretação da concordância, segundo o valor de kappa.

\begin{tabular}{c|c}
\hline \hline kappa & Concordância \\
\hline$<0,00$ & Ruim \\
\hline $0,00-0,20$ & Fraca \\
\hline $0,21-0,40$ & Sofrível \\
\hline $0,41-0,60$ & Regular \\
\hline $0,61-0,80$ & Boa \\
\hline $0,81-0,99$ & Ótima \\
\hline \hline
\end{tabular}

Fonte: Landis; Koch (1977)16. 
PINELLI, C.; LOFFREDO, L. C. M.; SERRA, M. C. Reprodutibilidade de um teste microbiológico para estreptococos do grupo mutans.

Pesq Odont Bras, v. 14, n. 1, p. 13-18, jan./mar. 2000.

TABELA 2 - Classificação dos resultados microbiológicos, segundo a recomendação do fabricante, considerando-se o conjunto dos "kits" 1, para ambos examinadores.

\begin{tabular}{c|c|c|c|c|c|c|c|c}
\hline \hline & \multicolumn{7}{|c}{ Examinador 1} \\
\hline & Escore & 1 & 2 & 3 & 4 & 5 & 6 & Total \\
\cline { 2 - 9 } & 1 & 1 & 1 & - & - & - & - & 2 \\
\cline { 2 - 9 } & 2 & - & 2 & - & - & - & - & 2 \\
\cline { 2 - 9 } Examinador 2 & 3 & - & 1 & 2 & - & - & - & 3 \\
\cline { 2 - 9 } & 4 & - & - & - & - & 1 & 1 & 2 \\
\cline { 2 - 9 } & 5 & - & - & - & 1 & 2 & - & 3 \\
\cline { 2 - 9 } & 6 & - & - & - & - & - & 2 & 2 \\
\cline { 2 - 8 } & Total & 1 & 4 & 2 & 1 & 3 & 3 & 14 \\
\hline \hline
\end{tabular}

po $=0,6429 ;$ pe $=0,1689 ; \kappa=0,5706 ; z=4,86$.

TABELA 3 - Classificação dos resultados microbiológicos, segundo a recomendação do fabricante, considerando-se o conjunto dos "kits" 2, para ambos examinadores.

\begin{tabular}{c|c|c|c|c|c|c|c|c}
\hline \hline & \multicolumn{7}{|c}{ Examinador 1} \\
\hline & Escore & 1 & 2 & 3 & 4 & 5 & 6 & Total \\
\cline { 2 - 9 } & 1 & - & - & 1 & - & - & - & 1 \\
\cline { 2 - 9 } & 2 & - & 1 & 2 & - & - & - & 3 \\
\cline { 2 - 9 } Examinador 2 & 3 & - & - & - & 2 & - & - & 2 \\
\cline { 2 - 9 } & 4 & - & - & - & 1 & 1 & 1 & 3 \\
\cline { 2 - 9 } & 5 & - & - & 1 & - & 1 & - & 2 \\
\cline { 2 - 8 } & 6 & - & - & - & 1 & - & 2 & 3 \\
\cline { 2 - 8 } & Total & - & 1 & 4 & 4 & 2 & 3 & 14 \\
\hline \hline
\end{tabular}

po $=0,3571 ;$ pe $=0,1837 ; \kappa=0,2125 ; z=3,74$.

\section{DISCUSSÃO}

Este trabalho descreve a utilização do produto CARITEST SM ${ }^{\circledR}$, em que 2 testes microbiológicos de lotes diferentes foram aplicados para um mesmo indivíduo. Os resultados obtidos, na classificação em escores de 1 a 6 , e na classificação em contagem microbiológica alta e baixa, foram distintos para lotes diferentes. Os valores de concordância, na classificação em 6 escores (Tabela 1), variaram de regular a sofrivel $(\kappa=0,57$ para o conjunto dos "kits" 1 e $\kappa=0,21$ para o conjunto dos "kits" 2). Maiores valores de concordância foram obtidos na classificação conforme a contagem microbiológica, alta e baixa, em que a variação foi de ótima a regu-
TABELA 4 - Classificação quanto à contagem microbiológica baixa e alta, considerando o conjunto dos "kits" 1 , para ambos examinadores.

\begin{tabular}{l|l|c|c|c}
\hline \hline & \multicolumn{4}{|c}{ Examinador 1 } \\
\hline \multirow{4}{*}{ Examinador 2 } & Contagem & Baixa & Alta & Total \\
\cline { 2 - 5 } & Baixa & 7 & - & 7 \\
\cline { 2 - 5 } & Alta & - & 7 & 7 \\
\cline { 2 - 5 } & Total & 7 & 7 & 14 \\
\hline \hline
\end{tabular}

po $=1,00 ;$ pe $=0,50 ; \kappa=1,00 ; z=3,74$.

TABELA 5 - Classificação quanto à contagem microbiológica baixa e alta, considerando o conjunto dos "kits" 2, para ambos examinadores.

\begin{tabular}{c|l|c|c|c}
\hline \hline & \multicolumn{4}{|c}{ Examinador 1 } \\
\hline \multirow{4}{*}{ Examinador 2 } & Contagem & Baixa & Alta & Total \\
\cline { 2 - 5 } & Baixa & 4 & 2 & 6 \\
\cline { 2 - 5 } & Alta & 1 & 7 & 8 \\
\cline { 2 - 5 } & Total & 5 & 9 & 14 \\
\hline \hline
\end{tabular}

po $=0,7857 ; p e=0,5204 ; \kappa=0,5532 ; z=2,09$.

lar $(\kappa=1,00$ para conjunto de "kits" 1 e $\kappa=0,55$ para conjunto de "kits" 2).

$O$ fato de ter sido observada uma menor concordância interexaminador, nas interpretações dos resultados conforme 6 escores, é justificável, pela subjetividade da classificação de 6 padrões, que possibilita a "valores" intermediários, que ora são vistos como pertencentes a uma categoria, ora a outra. Isto ocorre, devido às diferenças sutis entre as densidades de crescimento adjacentes do quadro de avaliação de resultados microbiológicos.

ADAIR et al. ${ }^{1}$, em 1994, ao estudarem a concordância interexaminador, mostraram que houve mais facilidade na interpretação dos resultados, quando se utilizaram 3 escores (pertencentes ao quadro de avaliação dos resultados do teste microbiológico BACTOTEST ${ }^{\circledR}$ ) ao invés dos 6 escores, propostos pelo outro teste, CARIESCREEN ${ }^{\circledR}$. O mesmo foi observado por MURTOMAA et al. ${ }^{19}$, em 1987: o uso de três escores, ao invés de quatro, promoveu o aumento da concordância, nas avaliações realizadas pela equipe de examinadores. No atual estudo, o valor de concordância foi maior para a classificação quanto à contagem microbiológica do que para a classificação em 6 escores, mostrando que há mais discordância ao se utilizar um número maior de níveis de classificação.

É correto que, na classificação segundo a contagem microbiológica alta e baixa, os valores te- 
PINELLI, C.; LOFFREDO, L. C. M.; SERRA, M. C. Reprodutibilidade de um teste microbiológico para estreptococos do grupo mutans.

Pesq Odont Bras, v. 14, n. 1, p. 13-18, jan./mar. 2000.

nham sido maiores para ambos "kits". Entretanto, os valores de concordância interexaminadores foram maiores para o "kit" 1 , nas duas situações de análise. Este fato pode ser justificado pelas condições de aplicação do produto, tendo sido melhores para o "kit" 1 do que para o "kit" 2. Além disso, acredita-se que existam fatores inerentes à fabricação do CARITEST-SM ${ }^{\circledR}$ capazes de afetar o resultado.

Durante a utilização do produto nesta pesquisa, verificou-se uma falta de padronização na embalagem dos tubos. Uns apresentaram só o lacre plástico, enquanto outros, além do lacre plástico, continham fita veda-rosca branca. Relatou-se também uma variação de volume do diluente tamponado, dentro de um mesmo "kit". Isso poderia implicar em diferentes diluições da bacitracina e afetar o crescimento das bactérias. Além disso, houve crescimento fúngico sobre duas lâminas de ágar, antes de expirado o prazo de validade, e estes tubos foram excluídos da pesquisa. KOGA, et al. ${ }^{12}$, 1995, também tiveram de excluir dois conjuntos de testes, do mesmo produto, porque, apesar de estarem dentro do prazo de validade, dois frascos da solução tampão encontravam-se contaminados e duas lâminas plásticas tiveram seus meios ressecados e descolados.

A falta de padronização do produto, somada aos valores distintos de concordância, encontrados no presente trabalho, suportam a afirmativa de que a diferença no lote e a avaliação em 6 escores resultaram numa variabilidade no resultado, podendo colaborar para níveis baixos de sensibilidade e especificidade, afetando a eficácia do teste microbiológico. Recomenda-se, portanto, a classificação dicotômica dos resultados, em alta e baixa contagem microbiológica, que favoreceu a reprodutibilidade interexaminador, possibilitando um resultado mais confiável.

É importante associar-se os resultados microbiológicos aos dados clínicos ${ }^{22}$, no momento da avaliação do risco ou da atividade de cárie dos pacientes submetidos ao tratamento odontológico, dadas as limitações apresentadas pelo produto. Novos trabalhos são necessários para comprovar a sua eficácia, visto que o teste microbiológico simplificado, que possui maior praticidade em relação ao método convencional, é considerado um dos parâmetros a serem avaliados no diagnóstico do risco de cárie.

\section{CONCLUSÕES}

Quando os resultados do teste microbiológico foram classificados, conforme a recomendação do fabricante, em escores de 1 a 6 , o índice de concordância interexaminador variou de regular $(\kappa=0,57)$ a sofrivel $(\kappa=0,21)$. Entretanto, quando se adotou a classificação conforme a contagem microbiológica alta e baixa, o índice de concordância variou de ótimo $(\kappa=1,00)$ a regular $(\kappa=0,55)$. Portanto, pelos valores obtidos para os índices de concordância, nas 2 situações de análise - na interpretação segundo recomendações do fabricante e na classificação quanto à contagem microbiológica, alta e baixa - alcançou-se maior reprodutibilidade diagnóstica na última situação.

\section{AGRADECIMENTOS}

Agradecemos à FAPESP (Processo 1997/ 10116-1 e 97/04921-9) pelo auxilio financeiro que possibilitou a realização desta pesquisa.

PINELLI, C.; LOFFREDO, L. C. M.; SERRA, M. C. Reproducibility of a simplified microbiological test for mutans streptococci. Pesq Odont Bras, v. 14, n. 1, p. 13-18, jan./mar. 2000.

The development of simple predictive microbiological tests allows targeting those children at highest risk for caries, even if they do not exhibit the disease activity. The aim of this study was to evaluate the reproducibility of CARITEST $\mathrm{SM}^{\circledR}$, a dip slide test for mutans streptococci. Fourteen subjects participated in this study. Saliva was stimulated by having the subject chew an inert gum and then collected until there was enough to perform two CARITEST SM ${ }^{\circledR}$ tests from different batches (set of kits 1 and set of kits 2) - applied twice for the same subject. Two calibrated examiners read each dip slide test once, by independent and blind means. The results were classified according to two criteria: scores from 1 to 6, recommended by the manufacturer's instructions, and scores according to high and low microbiological count. Kappa statistics was applied according to Light. The reproducibility results according to the 6 scores exhibited distinct values for the set of kits 1 and for the set of kits $2, \kappa=0.57$ and $\kappa=0.21$, respectively. The results according to high and low microbiological count were $\kappa=1.00$ for the set of kits 1 and $\kappa=0.55$ for the set of kits 2 . This study showed a variability in the results of both kits from different batches. Furthermore, there was greater interexaminer agreement when the results were classified according to high and low microbiological count.

UNITERMS: Microbiological tests; Saliva; Dental caries. 
PINELLI, C.; LOFFREDO, L. C. M.; SERRA, M. C. Reprodutibilidade de um teste microbiológico para estreptococos do grupo mutans.

Pesq Odont Bras, v. 14, n. 1, p. 13-18, jan./mar. 2000.

\section{REFERÊNCIAS BIBLIOGRÁFICAS}

1. ADAIR, S. M.; LEVERETT, D. H.; SHAFFER, C. L. Interexaminer agreement for readings of dip slide tests for salivary mutans streptococci and lactobacilli. Caries Res, v. 28, n. 2, p. 123-126, Mar./Apr. 1994.

2. ALALUUSUA, S.; SAVOLAINEN, J.; TUOMPO, H.; GRÖNROOS, L. Slide-scoring methods for estimation of Streptococcus mutans levels in saliva. Scand J Dent Res, v. 92, n. 2, p. 127-33, Apr. 1984.

3. ALALUUSUA, S.; RENKONEN, O-V. Streptococcus mutans establishment and dental caries experience in children from 2 to 4 years old. Scand J Dent Res, v. 91, n. 6, p. 453-457, Dec. 1983.

4. BLACK, G. V. A work on Operative Dentistry. Chicago, Medico-dental Publishing Company, 1908. v. 1, 144 p.

5. DODDS, M. W. J.; SUDDICK, R. P. Caries risk assessment for determination of focus and intensity of prevention in a dental school clinic. J Dent Educ, v. 59, n. 10, p. 945-956, Oct. 1995.

6. GOLD, O. G.; JORDAN, H. V.; VAN HOUTE, J. A selective medium for Streptococcus mutans. Archs Oral Biol, Oxford, v. 18, n. 11, p. 1357-1364, Nov. 1973.

7. IKEDA, T.; SANDHAM, H.J.; BRADLEY Jr., E. L. Changes in Streptococcus mutans and lactobacilli in plaque in relation to the initiation of dental caries in negro children. Arch Oral Biol, v. 18, n. 4, p. 555-566, Apr. 1973.

8. JENSEN, B.; BRATTHALL, D. A new method for the estimation of mutans streptococci in human saliva. J Dent Res, v. 68, n. 3, p. 468-471, Mar., 1989.

9. JORDAN, H. V.; LARAWAY, R.; SNIRCH, R.; MARMEL, M. A simplified diagnostic system for cultural detection and enumeration of Streptococcus mutans. J Dent Res, v. 66, n. 1, p. 57-61, Jan., 1987.

10. KEYES, P. H. Recent advances in dental caries research. bacteriological findings and biological implications. Int Dent J, v.12, n.4, p.443-67, Dec., 1962.

11. KLOCK, B.; KRASSE, B. Microbial and salivary conditions in 9- to 12-year-old children. Scand J Dent Res, v. 85, n. 1, p. 56-63, Jan., 1977.

12. KOGA, C. Y.; UNTERKIRCHER, C. S.; FANTINATO, V.; SHIMIZU, M. T.; JORGE, A. O. C. Testes de Atividade de Cárie. Avaliação de Diferentes Métodos. Rev Gaúcha Odontol, v. 43, n. 3, p. 141-144, maio/jun., 1995.

13. KÖHLER, B.; BRATTHALL, D. Practical method to facilitate estimation of Streptococcus mutans in saliva. J Clin Microbiol, v. 9, n. 5, p. 584-588, May, 1979.

14. KÖHLER, B.; PETTERSSON, B-M; BRATTHALL, D. Streptococcus mutans in plaque and saliva and the development of caries. Scand J Dent Res, v. 89, n. 1, p. 19-25, Feb. 1981

15. KRISTOFFERSSON, K.; AXELSSON, P.; BIRKHED, D.; BRATTHALL, D. Caries Prevalence, salivary Streptococcus mutans and dietary scores in 13-year-old Swedish schoolchildren. Community Dent Oral Epidemiol, v. 14, n. 4, p. 202-205, Aug., 1986.

16. LANDIS, J. R.; KOCH, G. G. The measurement of observer agreement for categorical data. Biometrics, v. 33, p. 159-174, 1977.

17. LIGHT, R. J. Measures of response agreement for quantitative data: some generalizations and alternatives. Psychol Bull, v. 76, p. 365-377, 1971.

18. MATSUKUBO, T.; OHTA, K.; MAKI, Y.; TAKEUCHI, M.; TAKAZOE, I. A semi-quantitative determination of Streptococcus mutans using its adherent ability in a selective medium. Caries Res, v. 15, n. 1, p. 40-45, Jan. 1981.

19. MURTOMAA, A.; MEURMAN, J. H.; RANTAMA, A.; LEVO, $\mathrm{S}$. Interexaminer variability in common ratings in reading Streptococcus mutans dip-slides with or without a microscope. Scand J Dent Res, v. 95, n. 2, p. 144-150, Apr. 1987.

20. NEWBRUN, E.; MATSUKUBO, T.; HOOVER, C. I.; GRAVES, R. C.; BROWN, A. T.; DISNEY, J. A.; BOHANNAN, H. M. Comparison of two screening tests for Streptococcus mutans and evaluation of their suitability for mass screenings and private practice. Community Dent Oral Epidemiol, v. 12, n. 5, p. 325-331, Oct. 1984.

21. ROSA, O. P. S. Niveis salivares de estreptococos mutans e lactobacilos e suscetibilidade à cárie dentária. CECADE News, v. 2, n. 1, p. 15-26, jan./abr., 1994.

22. VAN HOUTE, J. Microbiological predictors of caries risk. Adv Dent Res, v. 7, n. 2, p. 87-96, Aug. 1993.

23. VAN PALENSTEIN HELDERMAN, W. H.; MATEE, M. I. N.; VAN DER HOEVEN, J. S.; MIKX, F. H. M. Cariogenicity depends more on diet than the prevailing mutans streptococcal species. J Dent Res, v. 75, n. 1, p. 535-545, Jan. 1996.
Recebido para publicação em 02/08/99 Enviado para reformulação em 15/10/99 Aceito para publicação em 20/02/00 